LAW RENCE LIVERMORE N A T IO N A L LABORATORY

D. East

August 9, 2006 
This document was prepared as an account of work sponsored by an agency of the United States Government. Neither the United States Government nor the University of California nor any of their employees, makes any warranty, express or implied, or assumes any legal liability or responsibility for the accuracy, completeness, or usefulness of any information, apparatus, product, or process disclosed, or represents that its use would not infringe privately owned rights. Reference herein to any specific commercial product, process, or service by trade name, trademark, manufacturer, or otherwise, does not necessarily constitute or imply its endorsement, recommendation, or favoring by the United States Government or the University of California. The views and opinions of authors expressed herein do not necessarily state or reflect those of the United States Government or the University of California, and shall not be used for advertising or product endorsement purposes.

This work was performed under the auspices of the U.S. Department of Energy by University of California, Lawrence Livermore National Laboratory under Contract W-7405-Eng-48. 


\section{LC Computing Systems Summary}

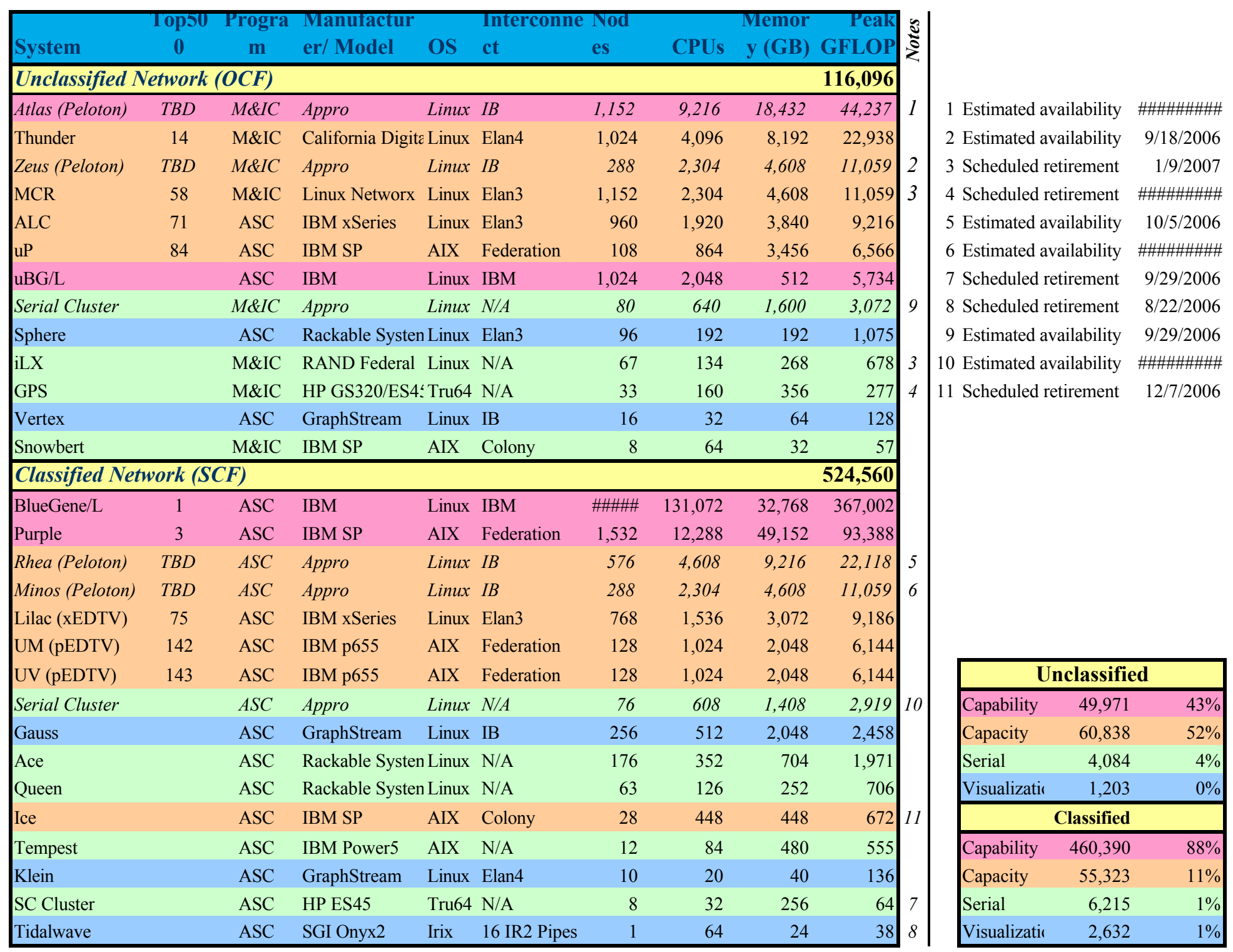




\begin{tabular}{|c|c|c|c|c|c|c|c|c|c|c|c|}
\hline System (Program) & Nodes & $\begin{array}{c}\text { CPUs/ } \\
\text { Node }\end{array}$ & $\mathrm{MHz}$ & FP & $\begin{array}{c}\text { Mem/ } \\
\text { Node }\end{array}$ & Nodes & $\begin{array}{c}\text { CPUs/ } \\
\text { Node }\end{array}$ & $\mathrm{MHz}$ & FP & $\begin{array}{r}\text { Mem/ } \\
\text { Node }\end{array}$ & Nodes \\
\hline Atlas & 1,152 & 8 & 2400 & 2 & 16 & & & & & & \\
\hline Thunder & 1,024 & 4 & 1400 & 4 & 8 & & & & & & \\
\hline Zeus & 288 & 8 & 2400 & 2 & 16 & & & & & & \\
\hline MCR & 1,152 & 2 & 2400 & 2 & 4 & & & & & & \\
\hline ALC & 960 & 2 & 2400 & 2 & 4 & & & & & & \\
\hline uPurple & 108 & 8 & 1900 & 4 & 32 & & & & & & \\
\hline uBG/L & 1,024 & 2 & 700 & 4 & 0.5 & & & & & & \\
\hline Serial Cluster & 60 & 8 & 2400 & 2 & 16 & 20 & 8 & 2400 & 2 & 32 & \\
\hline Sphere & 96 & 2 & 2800 & 2 & 2 & & & & & & \\
\hline iLX & 45 & 2 & 2400 & 2 & 4 & 22 & 2 & 2800 & 2 & 4 & \\
\hline GPS & 1 & 32 & 1000 & 2 & 32 & 16 & 4 & 1000 & 2 & 15.5 & 16 \\
\hline Vertex & 16 & 2 & 2000 & 2 & 4 & & & & & & \\
\hline Snowbert & 8 & 8 & 222 & 4 & 4 & & & & & & \\
\hline BlueGene/L & 65,536 & 2 & 700 & 4 & 0.5 & & & & & & \\
\hline Purple & 1,528 & 8 & 1900 & 4 & 32 & 4 & 16 & 1900 & 4 & 64 & \\
\hline Rhea & 576 & 8 & 2400 & 2 & 16 & & & & & & \\
\hline Minos & 288 & 8 & 2400 & 2 & 16 & & & & & & \\
\hline Lilac (xEDTV) & 730 & 2 & 3000 & 2 & 4 & 38 & 2 & 2800 & 2 & 4 & \\
\hline UM (pEDTV) & 128 & 8 & 1500 & 4 & 16 & & & & & & \\
\hline UV (pEDTV) & 128 & 8 & 1500 & 4 & 16 & & & & & & \\
\hline Serial Cluster & 64 & 8 & 2400 & 2 & 16 & 12 & 8 & 2400 & 2 & 32 & \\
\hline Gauss & 256 & 2 & 2400 & 2 & 8 & & & & & & \\
\hline Ace & 176 & 2 & 2800 & 2 & 4 & & & & & & \\
\hline Queen & 63 & 2 & 2800 & 2 & 4 & & & & & & \\
\hline GViz & 64 & 2 & 2800 & 2 & 4 & & & & & & \\
\hline Ice & 28 & 16 & 375 & 4 & 16 & & & & & & \\
\hline Tempest & 3 & 16 & 1650 & 4 & 64 & 9 & 4 & 1650 & 4 & 32 & \\
\hline Klein & 10 & 2 & 3400 & 2 & 4 & & & & & & \\
\hline SC Cluster & 8 & 4 & 1000 & 2 & 32 & & & & & & \\
\hline Tidalwave & 1 & 64 & 300 & 2 & 24 & & & & & & \\
\hline
\end{tabular}




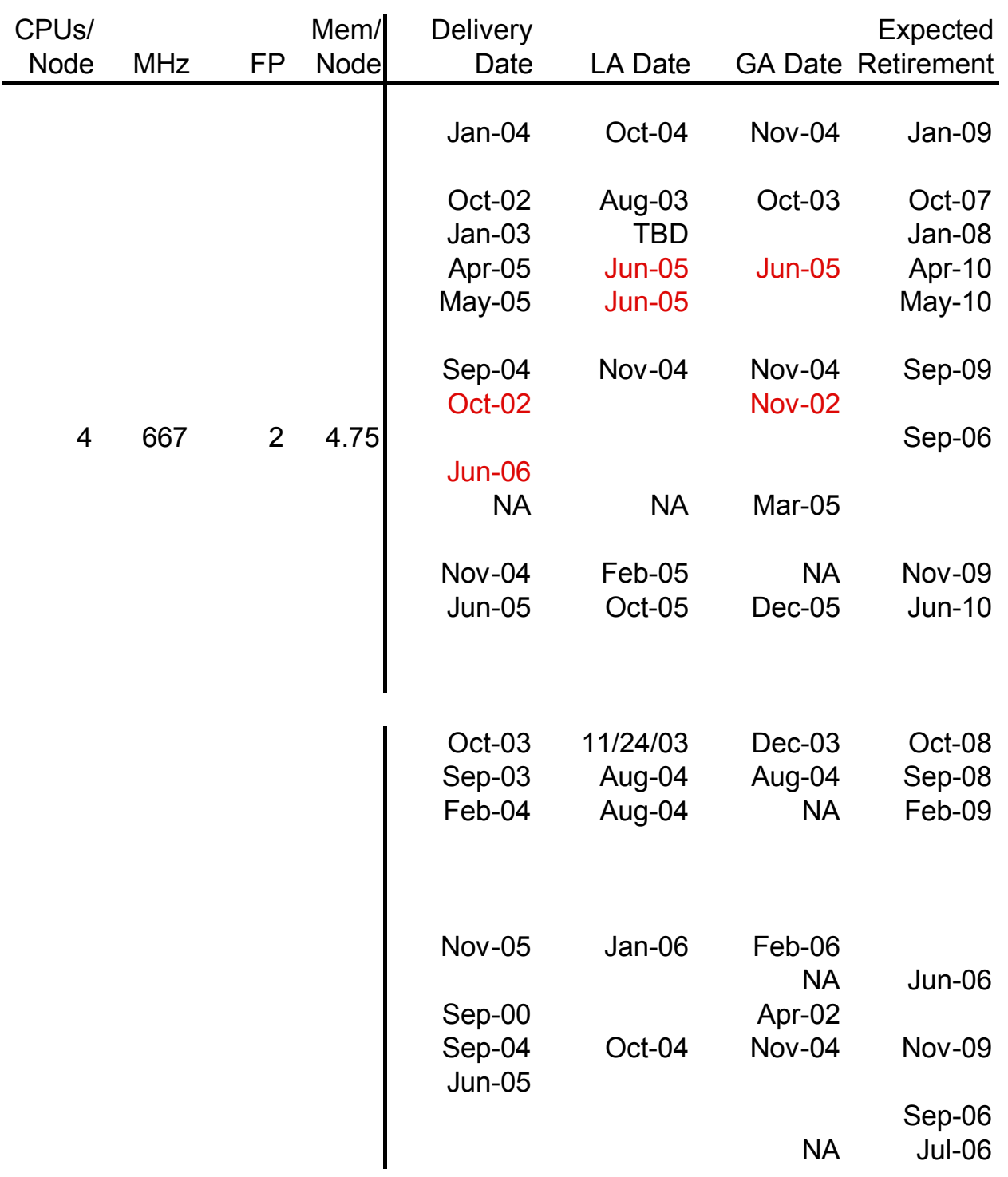




\begin{tabular}{|c|c|c|c|c|c|c|c|c|c|c|c|}
\hline \multirow[b]{2}{*}{ System } & \multicolumn{3}{|c|}{ CPUs/ } & \multicolumn{3}{|c|}{ Mem/ } & \multicolumn{2}{|l|}{ CPUs/ } & \multicolumn{3}{|c|}{ Mem/ } \\
\hline & Nodes & Node & $\mathrm{MHz}$ & FP & Node & Nodes & Node & $\mathrm{MHz}$ & FP & Node & Nodes \\
\hline Compass Cluster & 8 & 10 & 440 & 2 & 7 & & & & & & \\
\hline Forest Cluster & 5 & 9.2 & 625 & 2 & 8 & 1 & 8 & 500 & 2 & 16 & \\
\hline TC98 & 24 & 4 & 533 & 2 & 1 & & & & & & \\
\hline Sunbert & 1 & 24 & 250 & 2 & 16 & & & & & & \\
\hline Linux Cluster (LX) & 32 & 2 & 667 & 2 & 2 & 6 & 2 & 667 & 2 & 2 & \\
\hline Tera Cluster 98 & 24 & 4 & 533 & 2 & 1 & 12 & 2 & 500 & 2 & 1 & 6 \\
\hline Blue-Pacific (ASCI) & & & & & & & & & & & \\
\hline Sector S & 432 & 4 & 332 & 2 & 2.5 & 56 & 4 & 332 & 2 & 1.5 & \\
\hline Sector K & 488 & 4 & 332 & 2 & 1.5 & & & & & & \\
\hline Sector $Y$ & 488 & 4 & 332 & 2 & 1.5 & & & & & & \\
\hline Blue & 264 & 4 & 332 & 2 & 1.5 & & & & & & \\
\hline $\mathrm{TC} 2 \mathrm{~K}$ & 124 & 4 & 667 & 2 & 2 & 4 & 4 & 667 & 2 & 8 & \\
\hline Riptide & 1 & 48 & 250 & 2 & 36.6 & & & & & & \\
\hline GPS (partial) & & & & & & & & & & & 32 \\
\hline Edgewater & 1 & 40 & 300 & 2 & 17.6 & & & & & & \\
\hline Qbert & 2 & 10 & 613 & 2 & 12 & & & & & & \\
\hline SC Cluster (partial) & 32 & 4 & 667 & 2 & 4 & & & & & & \\
\hline Adelie & 128 & 2 & 2800 & 2 & 4 & & & & & & \\
\hline Emperor & 128 & 2 & 2800 & 2 & 4 & & & & & & \\
\hline Frost & 68 & 16 & 375 & 4 & 16 & & & & & & \\
\hline Whitecap & 1 & 96 & 400 & 2 & 96 & & & & & & \\
\hline PVC & 64 & 2 & 2400 & 2 & 2 & & & & & & \\
\hline White & 512 & 16 & 375 & 4 & 16 & & & & & & \\
\hline
\end{tabular}




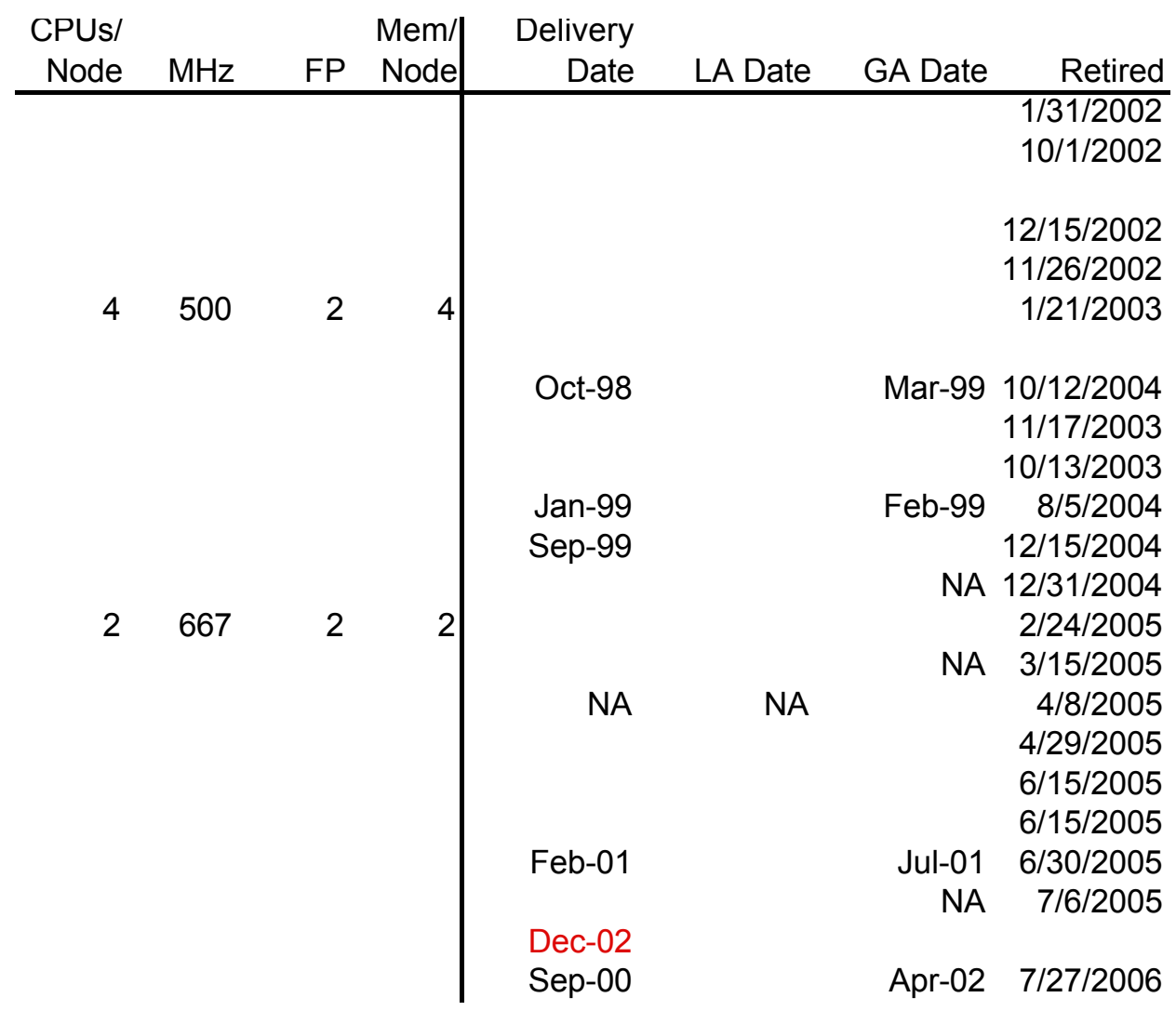

